\title{
Indian Sign Language Recognition System in Marathi Language Text
}

\author{
Prajakta Rokade \\ Dept. of Computer Engineering \\ Pimpri Chinchwad college of \\ Engineering, Pune, India
}

\author{
Archana Kadam \\ Dept. of Computer Engineering \\ Pimpri Chinchwad college of \\ Engineering, Pune, India
}

\author{
Dipti Shinde \\ Dept. of Computer Engineering \\ Pimpri Chinchwad College of \\ Engineering, Pune, India
}

\author{
Shalini Yadav \\ Dept. of Computer Engineering \\ Pimpri Chinchwad College of Engineering, Pune, \\ India
}

\author{
Neha Sali \\ Dept. of Computer Engineering \\ Pimpri Chinchwad College of Engineering, Pune, \\ India
}

\begin{abstract}
Sign languages are natural language that used to communicate with deaf and mute people.It is a significant way of communication between normal and deaf and dumb people,which does not require an interpreter. The main objective of this project is to develop a system that helps hearing and speech impaired people to convey their messages to ordinary people. There is much different sign language in the world. But the main focused of system is on Indian Sign Language (ISL) which is on the way of standardization in that the system will concentrated on hand gestures only. Hand gesture is very important part of the body for exchange ideas, messages, thoughts among deaf and dumb people. The proposed system will recognize the Indian hand sign language of words and sentences and translate the signs into Marathi text with images which have been extracted from the input videos. The process will be divided into three parts i.e. preprocessing, feature extraction, classification]. It will initially identify the gestures from Indian Sign language. Finally, the system processes that gesture to recognize character with the help of classification.
\end{abstract}

\section{Keywords}

Computer and information processing, Feature extraction, Gesture recognition, SVM, thinning algorithm

\section{1. INTRODUCTION}

Sign language is the basic means of communication for those with hearing and vocal disabilities. Deaf and dumb people have difficulty in their day to day lives.Sign language uses facial expressions,body movements and hand gestures for communication[5]. Sign language is not a universal language. Different countries have different sign languages. There are huge number of sign languages used worldwide. American sign language, Afghan sign language, Dutch sign language,Indian sign language are some of the examples of sign languages used. Signs in language are dependent on regional languages[5]. Deaf and dumb people need interpreter for conveying their messages to normal people and finding interpreters in day to day life is very difficult nowadays. The contribution of such a system will prove to be a boon for the deaf and dumb people. We aim to develop a system that would ease this difficulty in communication. Sign language consists of making shapes or movements with your hands with respect to the head or other body parts along with certain facial cues. A recognition system would thus have to identify specifically the head and hand orientation or movements, facial expression and even body pose.We propose the design for a basic yet extensible system that is able to recognize static and dynamic gestures of Sign Language.The system is able to perform in dynamic and minimally cluttered background with satisfactory result as it relies on skin color segmentation [3] to separate the gestures, i.e. focus on the hands and face. Since the lexicon under consideration (letters a-z) does not involve facial cues, we eliminate the face using Viola-Jones face detection followed by subtraction of the detected region[6]. We classify the gesture as static or dynamic by measuring the distance moved by the hand in subsequent frames. For static gestures, we use Zernike moments, a well known shape descriptor in image processing as shown in .For dynamic gestures we extract a curve feature vector which shows high accuracy in uniquely identifying paths .These feature vectors are then classified using pre-trained SVM classifiers. This helps map the gesture to a particular Marathi alphabet.

\section{EARLIER WORK}

Umme Santa had proposed a framework of Bangladeshi Hand Sign Language Recognition system for Bangla sentences from any input videos.[1] Dataset had been created which contained some video clips of some specific Bangla sentences as there was no available dataset for Bangla words and sentences[1].This paper discusses how to recognise hand gesture and convert it into Bangladeshi language[1].Firstly, input video was been converted into a set of video frames[1]. The hand was identified from the whole image by applying skin colour segmentation using $\mathrm{YCbCr}$ colour space[1]. Then the images were been converted into binary images. Binary image wea been cropped which contained only the skin portion[1]. Human face was been eliminated by calculating height of the input frames[1]. Local Binary Pattern algorithm was used for extracting features by applying it on the hand portion of the image[1]. Finally classification had been performed using support vector machine model.

\section{PROPOSED SYSTEM}

The Proposed work is to focus on the algorithms for classification of features extracted from hand gestures. Proposed model consist of four phases[2]:

A. Data acquisition : Self created database is used in proposed system. Database is generated by capturing videos of Indian sign Language. Videos are captured with the help of camera.

B. Preprocessing : The images extracted from the videos are preprocessed after the database collection. This step is necessary before extracting the features. It 
helps to neglect redundant and noisy data. Binary image will be generated after this phase.

C. Feature Extraction: The binary image obtained from the above step contains the hand-shape signifying the exact sign. The definite characteristics of the image need to be extracted to categorize the gesture.

D. Classification: The input to the the classification step will be the features extracted from the above step. The phases involved in classification are- training phase and testing phase. For this purpose, Support Vector Machine has been used.

E. Recognition.: The hand gesture is recognized and the meaning of it will be printed in marathi language as output of the system.

In the proposed model the thinning algorithm is used and also support vector machine (SVM) is used for classification of gesture. There are many features available for gesture recognition, but thinning algorithm is used.

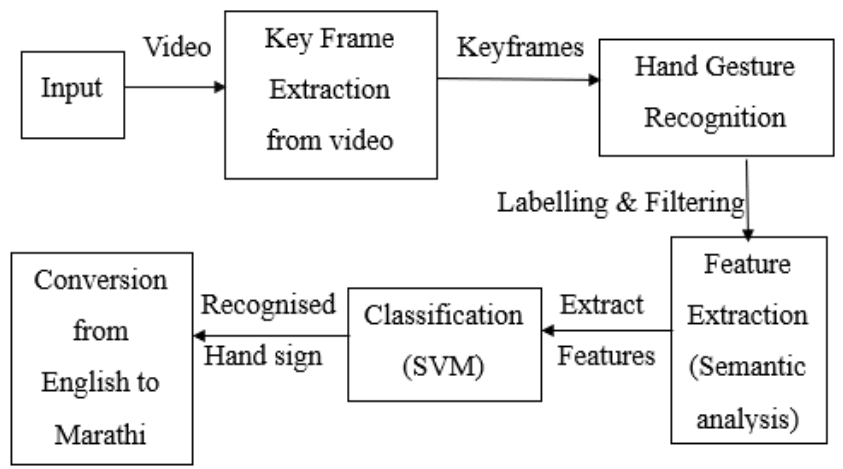

Fig.1 System Architecture

\subsection{Thinning Algorithm}

This algorithm is used for thinning binary image. Binary image by definition consists of only black and white pixels.

\section{Algorithm}

Assume black pixels are one and white pixels zero, and that the input image is a rectangular $\mathrm{N}$ by $\mathrm{M}$ array of ones and zeros.

The algorithm operates on all black pixels P1 that can have eight neighbors. The neighbors are, in order, arranged as

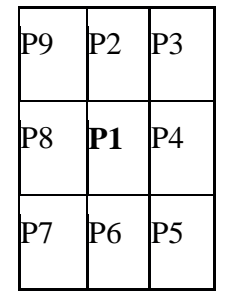

Obviously the boundary pixels of the image cannot have the full eight neighbors.

- Define $=$ the number of transitions from white to black, $(0 \rightarrow 1)$ in the sequence P2, P3, P4, P5, P6, P7, P8, $\mathrm{P} 9, \mathrm{P} 2$. (Note the extra P2 at the end - it is circular).

- Define $=$ The number of black pixel neighbors of P1. $(=\operatorname{sum}(\mathrm{P} 2 \ldots \mathrm{P} 9))$

\section{Step 1}

All pixels are tested and pixels satisfying all the following conditions (simultaneously) are just noted at this stage.
- (0) The pixel is black and has eight neighbors

- (1)

- (2) $\mathrm{A}(\mathrm{P} 1)=1$

- (3) At least one of P2 and P4 and P6 is white

- (4) At least one of P4 and P6 and P8 is white

After iterating over the image and collecting all the pixels satisfying all step 1 conditions, all these condition satisfying pixels are set to white.

\section{Step 2}

All pixels are again tested and pixels satisfying all the following conditions are just noted at this stage.

- (0) The pixel is black and has eight neighbors

- (1)

- (2) $\mathrm{A}(\mathrm{P} 1)=1$

- (3) At least one of P2 and P4 and P8 is white

- (4) At least one of P2 and P6 and P8 is white

After iterating over the image and collecting all the pixels satisfying all step 2 conditions, all these condition satisfying pixels are again set to white.

\section{Iteration}

If any pixels were set in this round of either step 1 or step 2, then all steps are repeated until no image pixels are so changed.

\subsection{Support Vector Machine}

Support vector machine is supervised learning algorithm which can be used for both regression and classification.SVM is regulated learning strategy[2].In this algorithm, system plots each data item as point in $\mathrm{n}$-dimensional space with the value of a particular coordinate[2].After that classification by finding the hyperplane that differentiate the two classes very well[2]. Support Vectors are simply the coordinates of individual observation. Support vectors are simply the best segregates the two classes. SVM is one of the best known methods in pattern classification and image classification. SVM classifier computationally costly and also runs slowly. SVM gives very accurate results[2]. It is designed to separate of a set of training images two different classes. (x1, y1),

$(x 2, y 2), \ldots,(x n, y n)$ where $x i$ in $R d, d$-dimensional feature space, and yi in $\{-1,+1\}$, the class label, with $i=1 . . n$ [1]. SVM builds the optimal separating hyper planes based on a kernel function (K). All images, of which feature vector lies on one side of the hyperplane, are belong to class -1 and the others are belong to class +1 . 


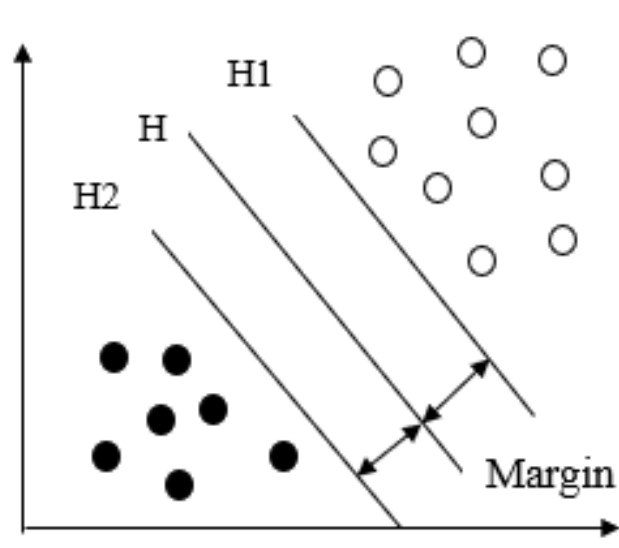

Fig.2 Support vector machine[2]

Table 1. Simple hand gestures

\begin{tabular}{|c|c|c|}
\hline Sr No. & Image & Word \\
\hline 1 & & I \\
\hline 2 & & Need \\
\hline 3 & & coffee \\
\hline 4 & & Open \\
\hline 5 & & Door \\
\hline 6 & & How \\
\hline
\end{tabular}

\begin{tabular}{|l|l|l|}
\hline 7 & Are \\
\hline 8 & & \\
\hline
\end{tabular}

\section{4. CONCLUSION}

Hand gesture recognition for real-life applications is very challenging because of its requirements on the robustness, accuracy and efficiency. In this work good comparative study has been adopted based on the methodology proposed using thinning algorithm and support vector machine. This will help to improving recognition accuracy of gesture and also recognize the active fingers names and position. In our proposed system, we process the input gesture in preprocessing phase, thinning method for feature extraction and finally using SVM for recognize the exact gesture name. It will recognize the Indian hand sign language of words and sentences and translate the signs into Marathi text. The effectiveness of the design in a real world situation has been demonstrated by a physical implementation of the system. This system will be developed by using python and it will be successfully implemented.

\section{ACKNOWLEDGEMENT}

We would particularly like to thank Prof. Archana Kadam for simulating discussion and for her valuable suggestion.

\section{REFERENCES}

[1] Umme Santa, Farzana Tazreen and Shayhan Ameen Chowdhury "Bangladeshi Hand Sign Language Recognition from Video" ,2017 20th International Conference of Computer and Information Technology (ICCIT)22-24 December, 2017

[2] Miss. Juhi Ekbote and Mrs. Mahasweta Joshi "Indian Sign Language Recognition Using ANN And SVM Classifier",2017 International Conference on Innovations in information Embedded and Communication Systems (ICIIECS)

[3] P. Subha Rajam and Dr. G. Balakrishnan "Real Time Indian Sign Language Recognition System to aid Deafdumb People".

[4] Geethu G Nath and Anu.V.S "Embedded Sign Language Interpreter System For Deaf and Dumb People" , 2017 International Conference on Innovations in information Embedded and Communication Systems (ICIIECS).

[5] Dr. Dharaskar Rajiv, Dr. Mr.Futane Pravin, "Hand Gesture Recognition System for numbers uses Thresholding", 2011.

[6] Priyal SP, Bora PK (2010). "A study on static hand gesture recognition using moments". In: Proceedings of international conference on signal processing and communications (SPCOM),

[7] Dong-Luong Dinh , Sung young Lee ,Tae Seong K, "Hand Number Gesture Recognition Using the Recognized Hand 
Parts in Depth Images", Springer Science Business Media New York 2014.

[8] Akanksha Singh, Saloni Arora Indian Sign Language Gesture Classification as Single or Double Handed Gesture Third International Conference on Image Intonation Processing, 2015.

[9] Anup Kumar, Mevin M. Domini, Sign Language Recognition 3rd In CI Condon Recent Advances in Information Technology I RAIT-2016.

[10] Wu CH, Lin CH (2013), "Depth-based hand gesture recognition for home appliance control". In: Proceedings of IEEE 17th international symposium on consumer electronics, pp 279-280
[11] Liu X, Fujimura K (2004). "Hand gesture recognition using depth data". In: Proceedings of IEEE international conference on automatic face and gesture recognition, pp 529-534

[12] Priyal SP, Bora PK (2010). "A study on static hand gesture recognition using moments". In: Proceedings of international conference on signal processing and communications (SPCOM), pp 1-5

[13] [13] Ren Z, Yuan J, Meng J, Zhang Z (2013). "Robust partbased hand gesture recognition using Kinect sensor". IEEE Trans. Trans Multimedia 15(5):1110-1120

[14] Barkocy A, Charkari NM (2011) Static hand gesture recognition of Persian sign numbers using thinning method. In: Proceedings of international conference on multimedia technology (ICMT), pp 6548-6551 